Wild type 1 poliovirus, the causative agent of poliomyelitis outbreak in Tajikistan where vaccine coverage dramatically decreased, was imported into Russia and was isolated from poliomyelitis cases and healthy migrants' from Tajikistan. We isolated WPV1 from three children who arrived in Russia from Tajikistan. The percentage of migrants' children who were seronegative to three types of poliovirus was 30 times higher than it was among resident Russian children. We isolated twice as many polioviruses from healthy migrants' children as from patients with acute flaccid paralysis in Russia.

The transmission of pathogenic revertant type 2 poliovirus from the unvaccinated paralytic patient to four healthy contacts in a hospital illustrated the emergence of VDPV with increased transmissibility. The vaccine-derived poliovirus of type 3 which displayed $1.1 \%$ nucleotide substitutions in the genomic region VP1 was isolated from a patient with VAPP who received two doses of oral polio vaccine (OPV). Another VAPP patient excreted polioviruses of types 1 and 2 after vaccination with OPV. A month later he stopped to excrete poliovirus of type 2, but he continued to excrete poliovirus of type 1 for more than 4 months. We also revealed the excretion of vaccine poliovirus of type 2 from VAPP patient till $105^{\text {th }}$ day after receiving four doses of oral polio vaccine. Vaccine poliovirus of type 3 was detected in the sample of unvaccinated 11-week-old patient with VAPP. We isolated the same poliovirus from the patient's sister who received 3 doses of inactivated polio vaccine and had high antibodies titers to polioviruses. She was the only possible source of poliovirus for the VAPP patient.

These data illustrate how poliovirus can persist in the population and confirm the possibility of limited spread of VDPV among well-immunized population. Reemergence of poliomyelitis can compromise Polio Eradication Initiative. It is indispensable to continue accurate surveillance and maintain polio free status of Russia as well as of other polio free countries.

\subsection{8} doi: $10.15789 / 2220-7619-2018-4-3.38$

\section{ROLE OF DIFFERENT TYPES OF ENTEROVIRUSES IN ETIOLOGY OF INFECTION ON CERTAIN TERRITORIES OF RUSSIA}

\section{N.I. Romanenkova, M.A. Bichurina, N.R. Rozaeva,}

\section{O.I. Kanaeva}

\section{St. Petersburg Pasteur Institute, St. Petersburg, Russia}

Epidemic peaks of enterovirus infection with the prevalence of different clinical forms of infection depend on different etiological factors. Outbreaks of hand, foot and mouth disease registered in the North-West of Russia in 2011-2012 were connected with Coxsackievirus A16 not detected previously in the region. The identification of two genetic variants closely related to strains isolated in France in 2010 and in Japan in 2011 suggested that Coxsackieviruses A16 implicated in these outbreaks had been brought to the NorthWest of Russia by two importation events. Echovirus 30 lineage which largely circulated in Russia in 2013 and caused outbreaks of meningitis in the North-West of Russia belonged to genotype $\mathrm{H}$ new for the region. Viruses implicated in outbreaks were closely related to the strains of genotype $\mathrm{H}$ detected in China in 2010-2013. Since earlier we detected in the country only Echovirus 30 of genotype Ec2 it is likely that Echovirus 30 of genotype $\mathrm{H}$ was imported into Russia from South-East Asia.

In 2016 Echovirus 30 of different variants of genotype $\mathrm{H}$ was implicated in epidemic peaks of enterovirus meningitis in Saratov and Kostroma regions. But a year later in Saratov region another type of enterovirus provoked a peak of enterovirus meningitis. It was Echovirus 18 which differed from viruses of the same type occasionally circulating in the North-West of Russia. In Murmansk and Leningrad regions in 2016 Coxsackieviruses A6 belonging to different genetic variants were the etiological factor of hand, foot and mouth disease. In Murmansk region and in the Komi Republic the cases of enterovirus infection with exanthema in 2017 were also connected mainly with Coxsackievirus A6. The strains of Coxsackievirus A6 identified in the North-West of Russia belonged to three sub-genotypes of pandemic genotype of Coxsackievirus A6.

Thus we detected Echoviruses 30 and 18 on territories where enterovirus meningitis was the leading form. On territories where enterovirus exanthema dominated the etiological factor of infection was Coxsackievirus A6. Our studies proved that surveillance of enterovirus infection aimed at acquiring new information about circulation of enteroviruses among population on different territories in different years is indispensable for prevention of propagation of enterovirus infection and for limitation of circulation of enteroviruses including the imported new serotypes/genotypes by means of using virological and molecular methods.

3.39

doi: 10.15789/2220-7619-2018-4-3.39

\section{MOLECULAR-GENETIC CHARACTERISTICS}

\section{OF THE COXSAKIE A10 ENTEROVIRUS THAT WAS} CIRCULATING IN THE CONSTITUENT ENTITIES \section{OF THE RUSSIAN FAR EAST}

\section{E.Yu. Sapega, L.V. Butakova, O.E. Trotsenko}

Khabarovsk Research Institute of Epidemiology and Microbiology, Khabarovsk, Russia

The 2017 epidemic season of enterovirus infection (EVI) was conditioned by circulation of Coxsackie A10 (CA10) in several constituent entities of the Far Eastern Federal District (FEFD) - the Khabarovsk, Primorsky Territories, Republic Sakha (Yakutia), Jewish Autonomous Region (JAR), Amursk and Magadan Territories. During the previous years the $C A 10$ was identified in individual cases and only in 2016 it was the cause of outbreaks in the Amursk city (the Khabarovsk Territory).

A total number of 90 strains of $C A 10$ were sequenced. A following phylogenetic analysis with the aid of BEAST program software and reference sequences obtained from the GenBank database was executed. A model of molecular clock was used to perform the evolutionary analysis.

Two genetic lines of $C A 10$ (A and B) were circulating in the observed constituent entities of the FEFD. The line A included enteroviruses (EV) isolated in the Republic Sakha (Yakutia) and Khabarovsk Region during 2016 as well as those that circulated in different regions of Russia in 2009-2013 and Europe in 2003-2010. The presented genetic variant was the source of the outbreaks in the Amursk city (the Khabarovsk Territory) registered in 2016. Divergence of the characteristics between Far Eastern and other Russian EV strains most likely took place in 2011 (CI: 2009-2012). The genetic line B was presented by $C A 10$ isolated in the FEFD in 2016-2017. The B-line strains isolated in the FEFD were divided into two clusters. First cluster was presented by the strains that circulated in the Khabarovsk, Primorsk, Magadan Territories and JAR in 2017 as well as those isolated in China in 2015. The most recent common ancestor (MRCA) for EV of the first cluster existed in 2013 (CI: 2012-2015). The second cluster included strains from the Republic Sakha (Yakutia) and Amur region isolated in 2016. However, this CA10 variant did not circulate in the constituent entities of the FEFD in 2017. 